Appl. Set-Valued Anal. Optim. 2 (2020), No. 3, pp. 271-272

Available online at http://asvao.biemdas.com

https://doi.org/10.23952/asvao.2.2020.3.01

\title{
EDITORIAL \\ A SPECIAL ISSUE DEDICATED TO HONG-KUN XU ON THE OCCASION OF HIS 6OTH BIRTHDAY, PART II
}

Professor Hong-Kun $\mathrm{Xu}$ is an outstanding mathematician and a leading expert in the theory of fixed points of nonlinear operators. This is the second part of the special issue on Trends in Fixed Points of Nonlinear Operators, which is dedicated to him on the occasion of his 60th birthday. This part contains seven papers contributed by leading experts as follows.

Based on a new iterative algorithm, Charles E. Chidume and Abubakar Adamu, in their paper "On split generalized mixed equality equilibrium and split equality fixed point problems," study the common solution of a split generalized mixed equality equilibrium problem and a split equality fixed point problem with quasi- $\phi$-nonexpansive mappings. A strong convergence theorem is proved without any compactness-type assumption on the mappings in $p$-uniformly convex and uniformly smooth real Banach spaces.

In the paper "Iterative algorithms for multiple-sets split feasibility problem in Banach spaces", Fenghui Wang considers a multiple-sets split feasibility problem. By converting it to a fixed point problem, two new iterative algorithms are introduced for solving the problem. Under some mild assumptions, the strong convergence of the proposed algorithms is obtained in Banach spaces.

Following [I.A. Rus, Gronwall lemma approach to the Hyers-Ulam-Rassias stability of an integral equation, in: Nonlinear Analysis and Variational Problems, Panos Pardalos, Themistocles M. Rassias, Akhtar A. Khan (ed.), pp. 147-152, Springer, 2009], Adrian Petruşel and Ioan A. Rus study the Ulam-Hyers stability of some ordinary differential equations based on Gronwall lemmas.

The primary focus of the paper, "Variants of the nonconvex minimization theorem and Caristi's fixed point theorem" by Maryam A. Alghamdi, Hadeel Z. Alzumi and Naseer Shahzad, is to obtain variants of the nonconvex minimization theorem and the Caristi's fixed point theorem in quasi-metric spaces. A generalized Ekeland's variational principle is also obtained in their paper.

Jingfang Xiao, Lu Huang and Yaqin Wan, in their paper "Strong convergence of modified inertial Halpern simultaneous algorithms for a finite family of demicontractive mappings," consider modified inertial Halpern simultaneous algorithms for a finite family of demicontractive mappings. Some strong convergence theorems under some suitable conditions are established in the framework of Hilbert spaces.

(C)2020 Applied Set-Valued Analysis and Optimizaton 
The goal of the work "A normal-subgradient algorithm for fixed point problems and quasiconvex equilibrium problems" by Le Hai Yen and Le Dung Muu is to find a common solution of a paramonotone quasiconvex equilibrium and a fixed point problem of nonexpansive mappings. They propose an algorithm, which is a combination of the projection method and the Krasnoselskii-Mann iteration scheme. Convergence of the algorithm is proved.

In recent years, the Douglas-Rachford algorithm received much attention due to its various applications in image recovery, signal processing, and machine learning. Yamin Wang and Haixia Zhang consider a viscosity Douglas-Rachford algorithm with multi-parameters, and establish its strong convergence under some mild conditions in the setting of Hilbert spaces.

Finally, we express our most sincere gratitude to all the authors who have contributed to this special issue and to the reviewers who helped us with their thorough reviews.

Adrian Petruşel

Department of Mathematics

Babeş-Bolyai University Cluj-Napoca, Romania

E-mail address: petrusel@math.ubbcluj.ro

Xiaolong Qin

Department of Mathematics

Hangzhou Normal University, Hangzhou, China

E-mail address: qxlxajh@163.com 